\title{
Two NFC Interaction Techniques for Quickly Exchanging Pictures between a Mobile Phone and a Computer
}

\author{
Khoovirajsingh Seewoonauth ${ }^{1}$, Enrico Rukzio ${ }^{1}$, Robert Hardy ${ }^{1}$, Paul Holleis ${ }^{2}$ \\ ${ }^{1}$ Computing Department, Lancaster University, UK, \{rukzio, hardyr\}@comp.lancs.ac.uk \\ ${ }_{2}^{2}$ DOCOMO Euro-Labs, Germany, holleis@docomolab-euro.com
}

\begin{abstract}
Uploading and downloading pictures between a mobile phone and a computer is still a very cumbersome process. Because of this, many users actually do not copy, move or backup their pictures onto another computer until the storage capacity of the mobile phone is reached. This paper presents the prototypes (and respective implementation details) of the touch \& connect and touch \& select interaction techniques. Both techniques allow the quick and easy exchange of pictures by touching the computer with the mobile device. The first interaction technique: touch \& connect, allows the user to touch a computer with their mobile phone in order to establish a Bluetooth connection and initiate a file transfer between the two devices. The second interaction technique: touch \& select, extends this concept and allows the selection of a specific picture or location on the computer screen by touching it with the mobile phone. The interaction techniques were implemented using Near Field Communication (NFC) tags attached to the computer and an NFC phone capable of reading those tags.
\end{abstract}

\section{Categories and Subject Descriptors}

H.5.2 [Information Interfaces and Presentation]: User Interfaces - Input devices and strategies; Prototyping.

\section{General Terms}

Human Factors, Design.

\section{Keywords}

Mobile, touch, interaction, display, picture sharing.

\section{INTRODUCTION}

Mobile phones are increasingly used for storing images, videos, documents and Personal Information Management (PIM) data. Furthermore, there is often the need to copy these files from the mobile phone to a computer or vice versa. This is typically a very cumbersome process because the user has to: find the right file, then select the method for transferring the file (e.g. Bluetooth), then perform a device discovery process, then select the target device. And after receiving the file on the other device, the user has to decide what to do with it. Consequently, this process is typically avoided by users which may have great consequences when the process concerns backing up important data [1].

This paper describes the implementation of two interaction techniques - touch \& connect and touch \& select - which were introduced in [2]. These simplify the transfer process dramatically by reducing the number of steps for copying a file. More importantly, touch \& select allows the mobile phone to act as a smart stylus as it can directly interact with a laptop or PC screen

Copyright is held by the author/owner(s). MobileHCI'09, September 15 - 18, 2009, Bonn, Germany. ACM 978-1-60558-281-8 through touch.

Although touch \& connect and touch \& select can be used for interaction with any kind of display, this demonstration focuses on the usage of a laptop, such a device was used for the implementation of the prototype.

Figure 1 illustrates how touch \& connect supports copying a file from a mobile phone to a laptop and vice versa. When uploading a file from the phone to the laptop, the user simply selects the file on the phone and touches the laptop in order to start the transmission. The file is then copied to the laptop.

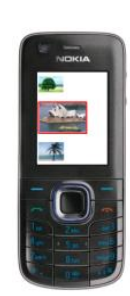

1. Select

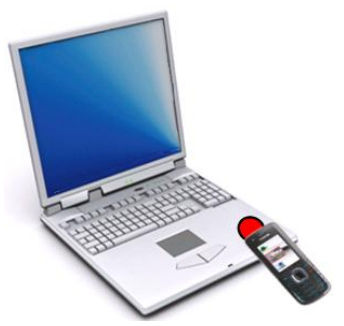

2. Touch the laptop

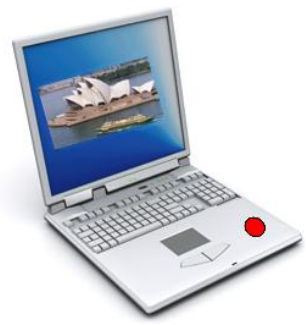

3. Image is transferred to the laptop
Figure 1. Copying a file from a phone using touch \& connect.

Reversely, the user can download a file to the phone by selecting it on the laptop via the mouse/touchpad, then touching the laptop with the phone in order to copy the file to it (Figure 2).

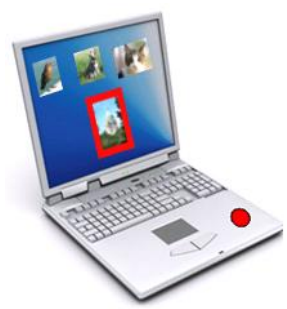

1. Select

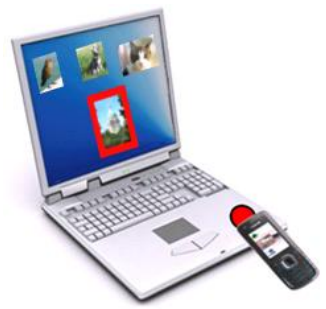

2. Touch the laptop

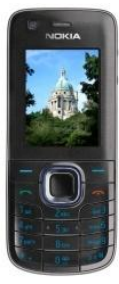

3. Image is transferred to the phone
Figure 2. Copying a file to a phone using touch \& connect.

In touch \& connect, the user does not need to define the method for transferring the file, does not need to wait for the end of the device discovery process, and does not need to select the target device. All this information is gathered when touching the laptop with the mobile phone.

Figure 3 illustrates how touch \& select, an extension of touch \& connect, can be used to transfer files between a mobile phone and a laptop. When uploading a file to the laptop, the user has to select it on the mobile phone, then the mobile phone can be used to touch a location on the laptop screen in order to copy it to this place. 


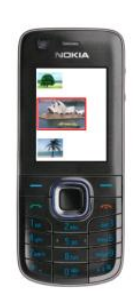

1. Select

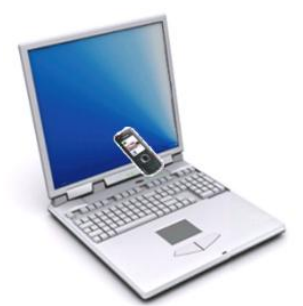

2. Touch laptop screen

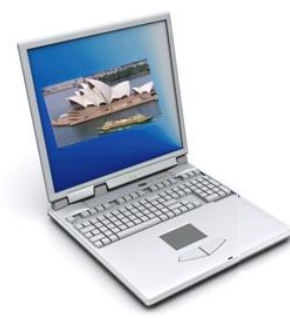

3. Image is transferred to the laptop
Figure 3. Copying a file from a phone using touch \& select.

The user can download a file to the phone by touching the display of the laptop with the mobile phone on the position at which the file is displayed (Figure 4).

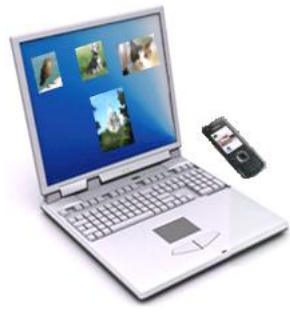

1. Find the image

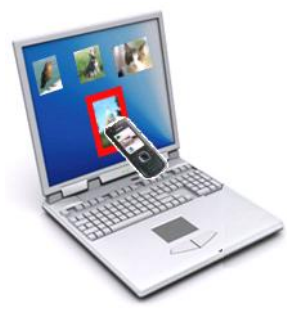

2. Touch the image

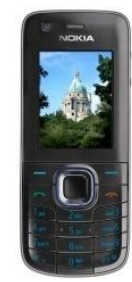

3. Image is transferred to the phone
Figure 4. Copying a file to a phone using touch \& select.

Touch \& select provides all the advantages of touch \& connect; however, it additionally allows the direct interaction with objects, files and folders on the laptop screen.

The next section relates our work to existing approaches. Following this, we report the implementation of the touch \& connect and touch \& select prototypes using Near Field Communication (NFC) technology. The two prototypes were evaluated in a comparative user study in order to show the advantages and disadvantage of them when compared with standard Bluetooth based file transfer. Finally, the paper discusses our findings and provides an outlook on future work.

\section{RELATED WORK}

Mobile phones are commonly used for taking pictures, receiving MMS, recording videos or storing files. These files have often to be transferred to a computer when one wants to view them on a large screen, they should be attached to an email and when making a backup. An USB cable or Bluetooth are currently mainly used when transferring those files [1]. Many users avoid copying files as an USB cable is often not at hand and Bluetooth requires often a complicated pairing process.

Many research projects have already focused on mobile interaction with displays, computers and objects (see [3] for a corresponding overview). According to previous research, most users prefer touch-based interactions over pointing or indirect interaction when within near proximity to the devices [4]. The reason for this is that people are very familiar with touch based interactions and because of the speed and simplicity of touching an object when in close proximity.

The concept of touching an object with a mobile device has been widely investigated in the last years. Most implementations are based on RFID/NFC technology. Want et al. were among the first who connected an RFID reader to a mobile device and equipped objects in the environment with RFID tags [5].

Near Field Communication (NFC) phones are able to read from and write information to RFID/NFC tags and were used for the implementation of the two presented interaction techniques. First NFC phones are already available and it is predicted that several hundred million NFC equipped mobile phones will be used in 2013 [6]. The basic idea of touch \& connect and its implementation using NFC/RFID technology has already been described by ECMA International in 2004 [7] and is since 2007 also part of the Bluetooth 2.1 standard but was not applied for picture sharing so far.

Reilly et al. developed a system in which they augmented a paper map with a mesh of RFID tags [8]. Through this, it was possible to touch any position on the map in order to select a point of interest. The touch \& interact system, presented by Hardy and Rukzio, used the same approach using a projection instead of a paper map [9]. With this approach, it was now possible to change the information shown on the projection according to the interaction of the user. When a user touched the display at a certain position, both the projection and mobile phone display changed accordingly. The touch \& select interaction technique presented in this paper is very similar except that a laptop display is used to overcome several disadvantages of using a projectionbased system. The most important disadvantage of touch \& interact is the occlusion of the projection when the user touches the screen with the mobile phone.

Both Pick-and-Drop from Rekimoto [10] and the two interaction techniques presented in this paper share the concept of interaction between a mobile device and a display. However, Pick-and-Drop requires an additional pen which is used as the interaction device whereas our approach eliminates the use of a pen as both devices touch each other directly.

Further work related to the touch \& select interaction technique is the usage of interactive surfaces like Microsoft Surface which supports interaction with mobile devices. It is possible to put a mobile device on a Microsoft Surface and drag \& drop photos in order to copy them to or from the mobile device. The difference between Microsoft Surfaces and touch \& select is that, with Microsoft Surfaces, the user primarily interacts with the interactive surface, and the mobile phone acts primarily as a data container. In touch \& select, the mobile phone is the primary interaction device and both displays (mobile phone and laptop screen) are used in parallel.

\section{PROTOTYPES AND DEMONSTRATION}

This section discusses the implementation of the touch \& connect and touch \& select prototypes. For these interactions, we focused on the implementation of a photo exchanging and browsing application as we see this scenario as the most common for connecting a mobile phone with a computer. These prototypes were developed in order to compare task completion time and usability of these two new interaction techniques with the currently used conventional Bluetooth-based file transfer. Both prototypes provide functions for uploading (from the mobile phone to the laptop) and downloading (from the laptop to the mobile phone) pictures.

We developed two photo browsing applications (Figure 5), one for the mobile phone and one for the laptop. We developed these two rather than adapting existing applications to support the new 
interaction techniques touch \& connect and touch \& select. This allowed us to keep a consistent interface on the mobile phone and on the laptop for the comparative user study. Moreover, it was then simple to add logging features to our own applications through which it was easily possible to control the user study and to automatically gather information like task completion time.

The mobile phone photo browser application was designed to look very much like the standard photo browser of the Nokia 6131 NFC phone (which was used in the study). In both applications, the user was able to browse through the available pictures using the directional keys (phone and laptop) or the mouse (laptop) in order to enlarge pictures and to select pictures in order to see available options.

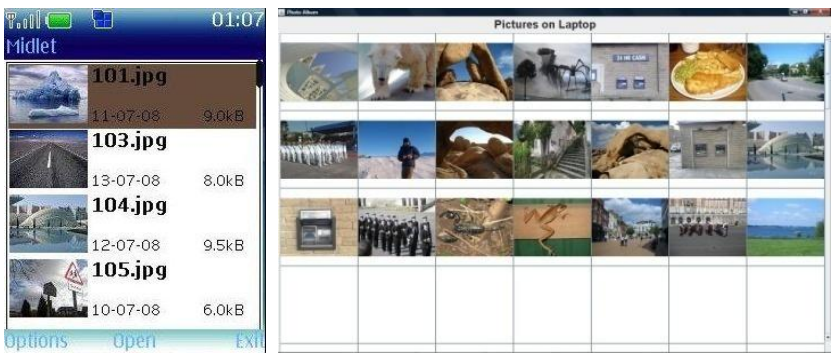

Figure 5. Photo browsing application on mobile phone (left) and laptop (right)

In the subsequent sections, the usage of the two different implemented interaction techniques touch \& connect and touch \& select will be discussed.

\subsection{Touch \& Connect}

A yellow sticky note was used to indicate the location of the NFC tag so that the participants knew where to touch.

To upload a picture, the user first selects a picture on the phone, then chooses upload. The mobile phone informs the user (Figure 6 left) to touch the yellow tag on the laptop in order to upload the image (Figure 6 middle). The picture is then copied and displayed on the laptop (Figure 6 right).

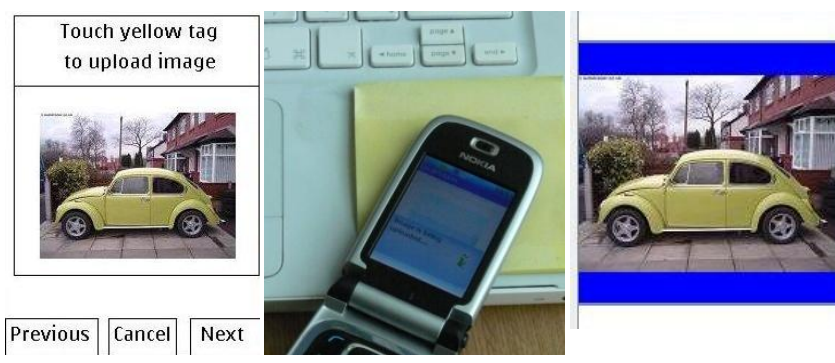

Figure 6. Uploading a picture from the phone to the laptop with touch \& connect

In order to download an image, it is selected using the mouse in the picture browsing application on the laptop, then Send To and Mobile phone via yellow tag are selected (Figure 7 left). The user can then touch the yellow tag on the laptop (Figure 7 middle) to copy the image to the phone (Figure 7 right).
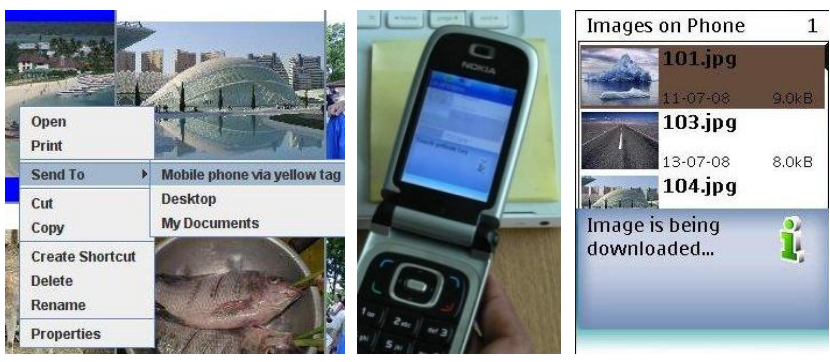

Figure 7. Downloading a picture from the phone to the laptop with touch \& connect

\subsection{Touch \& Select}

To upload a picture, the user first selects a picture on the phone and chooses upload. The mobile phone then informs the user that an empty square on the display can be touched in order to upload the image (Figure 8 left). When a user touches the display (Figure 8 middle), the corresponding picture is uploaded and shown on the screen of the laptop (Figure 8 right).

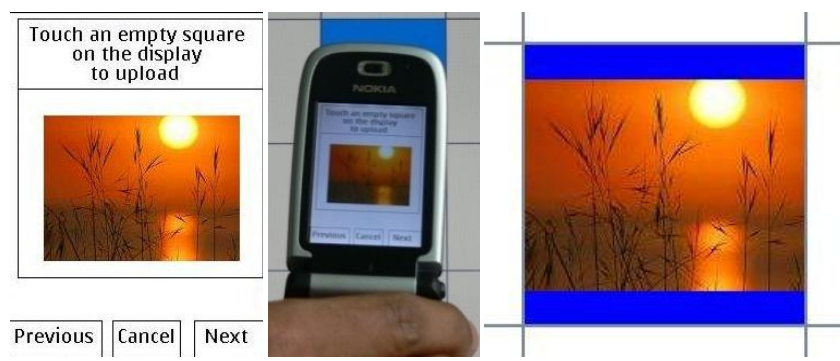

Figure 8. Uploading a picture from the phone to the laptop with touch \& select

In order to download an image, the user switches to download mode on the mobile phone (Figure 9 left) and selects the picture on the laptop by touching it with the mobile phone (Figure 9 middle). The picture is then copied to the mobile phone (Figure 9 right) and displayed there.

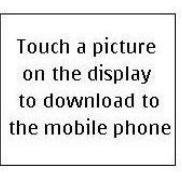

$$
\text { Cancel }
$$
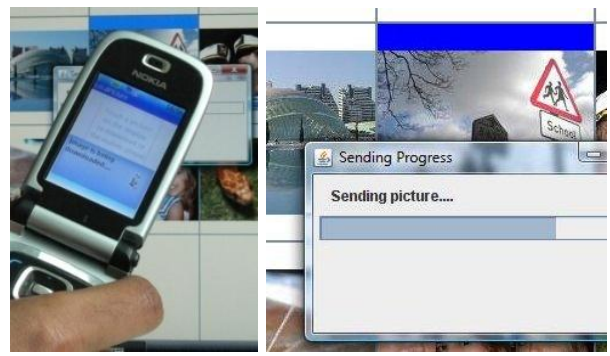

Figure 9. Downloading a picture from the phone to the laptop with touch \& select

\section{IMPLEMENTATION}

A MacBook laptop and a Nokia 6131 NFC phone were used for the implementation of the prototypes. Java SE and the Bluecove library (http://code.google.com/p/bluecove/) were used to implement the picture browsing application on the laptop and its communication with the mobile phone. On the phone, Java ME and the Java APIs for Bluetooth (JSR 82) were used. The implementations specific to each interaction technique will now be discussed. 


\subsection{Touch \& Connect}

For touch \& connect, a Near Field Communication tag from toptunniste.fi (Trikker BT43) was attached to the laptop as shown in Figure 6 or 7. This tag stores the Bluetooth MAC address of the corresponding laptop through which it is possible to establish a spontaneous connection without the need for a device discovery process. The two devices communicate with each other over this Bluetooth link to exchange the pictures.

\subsection{Touch \& Select}

For touch \& select, the back of the laptop display was augmented with a mesh of 7 x 4 NFC tags (Trikker BT43) as shown in Figure 10 (left). Each tag stored its location (Figure 10 middle) and the Bluetooth MAC address of the laptop. When touching the front of the laptop display (Figure 10 right), the NFC phone is able to read the stored information from the tags which were attached the rear of the display. It then connects with the laptop using the Bluetooth MAC address (stored in every NFC tag) and submits the coordinates of the tag, e.g. $(2,2)$, to the laptop. Once a connection is set up, the picture browsing application on the laptop can receive messages indicating which picture or empty square was selected and responds accordingly.

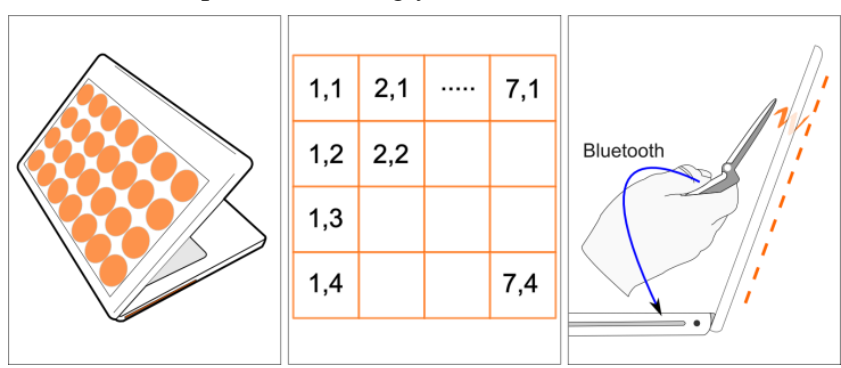

Figure 10. Implementation of touch \& select

The MacBook was used because its plastic casing ensures a good connection between the NFC phone touching the front of the display and the NFC tags attached to the back of the display. One could imagine such NFC functionalities to be embedded in many commercial displays in the future.

\section{EVALUATION}

The authors conducted a comparative study in which the two new interaction techniques were compared with the currently used Bluetooth-based approach in order to analyze and compare: task completion time, error rate, usability satisfaction, task load and user preferences [2]. This study showed that touch \& select is significantly better than touch \& connect, which is again significantly better than the currently used Bluetooth-based method. When focusing on task completion time for uploading and downloading pictures, the conventional Bluetooth-based implementation was $44 \%$ slower than touch \& connect and $76 \%$ slower than touch \& select. When asked for their preferences for uploading and downloading, 71\% saw touch \& select as their first choice but only $18 \%$ saw touch \& connect and $11 \%$ standard Bluetooth as their first choice.

\section{DISCUSSION AND OUTLOOK}

This paper presents the concept and implementation of the novel interaction technique: touch \& select, in which a user can interact with objects or locations on the laptop display by touching them with their mobile phone. The advantage of this is the ability to directly interact with displayed objects without requiring a complicated device coupling and selection process.
Furthermore, we present an implementation for the touch \& connect interaction technique in which the user is not able to select objects displayed on the laptop screen using the mobile phone. But the device coupling process and the initiation of a file transfer is easily done through touching a tag on the laptop's armrest.

The NFC-based implementations presented in the paper have the disadvantage that such mobile phones are not widely available everywhere. However, it has the potential to be integrated in most future phones [6]. A disadvantage of touch \& select is the limited input resolution defined by the currently relatively large NFC tags and the size of the mobile phone. Several solutions for this, such as showing an enlarged version of the selected area on the laptop display have been discussed by Hardy and Rukzio [9], could be applied for touch \& select.

In our future work, we will focus on new interaction techniques that will use touch screens provided by some mobile phones as well as the laptop. In addition, we will focus on application areas other than photo browsing such as synchronization of a mobile phone with a laptop, general file transfer, and using touch \& select and touch \& connect for interactions with electronic picture frames and larger public displays.

\section{ACKNOWLEDGEMENT}

The presented research was conducted in the context of the Multitag project which is funded by DOCOMO Euro-Labs.

\section{REFERENCES}

[1] Puikkonen, A. M., Häkkilä, J., Ballagas, R., Mäntyjärvi, J. Practices in Creating Videos with Mobile Phones. In Mobile HCI 2009.

[2] Seewoonauth, K., Rukzio, E., Hardy, R., Holleis, P. Touch \& Connect and Touch \& Select: Interacting with a Computer by Touching it with a Mobile Phone. In Mobile HCI 2009.

[3] Rukzio, E. Physical Mobile Interactions: Mobile Devices as Pervasive Mediators for Interactions with the Real World. PhD Dissertation. University of Munich. 2007.

[4] Rukzio, E., Leichtenstern, K., Callaghan, V., Holleis, P., Schmidt, A., Chin, J. An Experimental Comparison of Physical Mobile Interaction Techniques: Touching, Pointing and Scanning. In Ubicomp'06, 87-104, 2006

[5] Want, R., Fishkin, K. P., Gujar, A., Harrison, B. L. Bridging Physical and Virtual Worlds with Electronic Tags. In CHI'99, 370-37, 1999

[6] Juniper Predicts 700 Million NFC Cell Phones by 2013. In ContactlessNews. September 10, 2008._ECMA International. Near Field Communication White paper. Ecma/TC32TG19/2004/1. http://www.ecmainternational.org/activities/Communications/2004tg19001.pdf

[7] Reilly, D., Rodgers, M., Argue, R., Nunes, M., Inkpen, K. Marked-up Maps: Combining Paper Maps and Electronic Information Resources. In Personal and Ubiquitous Computing, 10 (4), 215-226, 2006.

[8] Hardy, R., Rukzio, E. Touch \& Interact: Touch-based Interaction of Mobile Phones with Displays. In MobileHCI'08, 245-254, 2008.

[9] Rekimoto, J. Pick-and-drop: a Direct Manipulation Technique for Multiple Computer Environments. In UIST'97, 31-39, 1997. 